\title{
Criterion adjustment and probability matching
}

\author{
EWART A. C. THOMAS \\ Stanford University, Stanford, California 94305
}

\begin{abstract}
Probability matching is contraindicated by the data of individual subjects (Dusoir, 1974) and by group data (Creelman \& Donaldson, 1968); in general, response frequencies are not as extreme (i.e., distant from one-half) as the corresponding stimulus frequencies. This deviation from probability matching can be accounted for by at least two versions of an error-correcting model in which the subject's decision criterion may shift only after an error, but not by a model which permits criterion shifts after correct responses. One version of the error-correcting model allows us to specify the dependence of response frequency on pay off magnitude and on signal strength. The deviation from matching is consistent also with a model that assumes that, on each trial, the criterion shifts in the direction of the sensory information, regardless of response accuracy. This model is thus applicable to situations in which trial-by-trial feedback is omitted.
\end{abstract}

When a subject's performance in a detection or recognition task is analyzed by means of the theory of signal detectability, at least two classes of issues arise concerning the decision criterion used by the subject. In the first class, it is assumed that the criterion is the same on all trials, and the issues concern how this static criterion is determined and how it varies across experimental conditions having different payoff schedules and/or signal strength. In the second class, it is assumed that the criterion may vary over trials, and the issues center around the nature of this (dynamic) process. These two classes of issues overlap when one regards the "static" criterion (of the first class) as the mean value of some stationary stochastic process. This seems to be the case when the error-correcting model (see, e.g., Kac, 1962) is the stochastic process used to account for the particular rule for determining the "static" criterion known as probability matching (see, e.g., Parks, 1966).

Let us consider a yes/no detection task in which, on each trial, signal-plus-noise $\left(s_{1}\right)$ is presented with probability $\pi$ and noise $\left(s_{2}\right)$ is presented with probability $1-\pi$. The matching hypothesis states that, when the payoff matrix is symmetric (i.e., payoff depends only on response accuracy), the frequency with which the subject reports the signal, $\mathrm{P}\left(\mathrm{r}_{1}\right)$, equals $\pi$ (Creelman \& Donaldson, 1968; Parks, 1966; Thomas \& Legge, 1970). Very recently, Dorfman, Saslow, and Simpson (1975) and Dusoir (1974) have statistically tested the matching hypothesis, using data from individual subjects, and the first of these studies has shown that this hypothesis is in error. The data of Dusoir show that, for most subjects, $P\left(r_{1}\right)$ is significantly less than $\pi$ for $\pi$ greater than .5. The same conclusion can be drawn from the group data of Creelman and Donaldson, which show that, when

This work was supported by Grant GB43275 from the National Science Foundation. I am grateful to a referee for helpful comments. payoffs are low, the decision criteria are less extreme than the values predicted by probability matching. On the other hand, Thomas (1973, Table 1) suggests (without a statistical test) that the group data of Dorfman and Biderman (1971) are consistent with the matching hypothesis.

An error-correcting model is one in which the decision criterion shifts only after the subject makes an error, either (a) with probability 1 and by an amount $\Delta_{\mathbf{i j}}$ which depends on the payoff associated with response $i$ and the stimulus $j$, or (b) with probability $p_{i j}$ and by an amount $\Delta$. The direction of the shift is such as to reduce the probability of an incorrect response. It has been shown that the first alternative, the deterministic shift model, predicts probability matching when $\Delta_{12}=\Delta_{21}$ (Dorfman et al., 1975; Kac, 1962; Norman, 1972), as does the probabilistic shift model when $p_{12}=p_{21}$ (Thomas, 1973).

The error-correcting model is applicable only to experiments in which trial-by-trial feedback is given to the subject, since it is only in these situations that criterion adjustment can depend on response accuracy. Such feedback was given in the studies by Creelman and Donaldson (1968) and Dorfman et al. (1975), but not in the Dusoir (1974) study. To account for data obtained without feedback, we shall consider a model in which criterion adjustment does not depend on response accuracy.

\section{EXPERIMENTS WITH FEEDBACK}

The error-correcting model is applicable to these situations, and the issue we shall consider is what modifications of the model are sufficient to account for the observed deviation from probability matching, and the observed dependence of the "static" criterion on payoffs and signal strength. In this note, we consider only the probabilistic shift alternative of the model. 


\section{Model I}

One modification is the suggestion that, even when the payoff matrix is symmetric, $p_{12} \geqslant p_{21}$ if and only if $\pi \geqslant 1 / 2$. This amounts to saying that, given an error, the probability of a criterion shift is greater if the stimulus is the less likely one. That is, if the subject is given immediate feedback following an error, the less frequent reinforcing event is the more potent. When stated in these terms, the modification seems reasonable. To show that it can account for some deviations from probability matching, we may note that (Thomas, 1973, Equation 13),

$$
\frac{P\left(r_{1} \mid s_{2}\right)}{1-P\left(r_{1} \mid s_{1}\right)}=\frac{p_{21} \pi}{p_{12}(1-\pi)} \leqslant \frac{\pi}{1-\pi} \text { if } p_{12} \geqslant p_{21} .
$$

Therefore, if $\pi \geqslant 1 / 2$ implies $\mathrm{p}_{12} \geqslant \mathrm{p}_{21}$, we have, by rearranging Equation 1, that when $\pi \geqslant 1 / 2$,

$$
\mathrm{P}\left(\mathrm{r}_{1}\right)=\pi \mathrm{P}\left(\mathrm{r}_{1} \mid \mathrm{s}_{1}\right)+(1-\pi) \mathrm{P}\left(\mathrm{r}_{1} \mid \mathrm{s}_{2}\right) \leqslant \pi .
$$

However, this modification does not allow us to specify how the mean criterion value depends on signal strength.

\section{Model II}

A second modification of the error-correcting model is obtained by allowing the criterion to shift after correct responses with probability $p_{11}$ or $p_{22}$, depending of whether the stimulus is $s_{1}$ or $s_{2}$, respectively. The direction of the shift is such as to increase the probability of a correct response (cf. Dorfman \& Biderman, 1971, for the deterministic shift alternative). When the payoff matrix is symmetric, we assume $p_{11}=p_{22}$ and $p_{12}=p_{21}$, and let $a=p_{22} / p_{12}$. For this case, Thomas (1973, Equation 4 and Theorem 2.5) has shown that the hit and false-alarm rates satisfy

$$
\frac{\mathrm{P}\left(\mathrm{r}_{1} \mid \mathrm{s}_{2}\right)+\mathrm{a}\left[1-\mathrm{P}\left(\mathrm{r}_{1} \mid \mathrm{s}_{2}\right)\right]}{1-\mathrm{P}\left(\mathrm{r}_{1} \mid \mathrm{s}_{1}\right)+\mathrm{aP}\left(\mathrm{r}_{1} \mid \mathrm{s}_{1}\right)}=\frac{\pi}{1-\pi} .
$$

It is easily seen that the left-hand side of Equation 3 is greater than $P\left(r_{1} \mid s_{2}\right) /\left[1-P\left(r_{1} \mid s_{1}\right)\right]$ if and only if the latter ratio is less than 1 . Therefore, to account for the data that $\mathrm{P}\left(\mathrm{r}_{1}\right) \leqslant \pi$ when $\pi \geqslant 1 / 2$, we need to assume that, when $\pi \geqslant 1 / 2, P\left(r_{1} \mid s_{2}\right)$ is less than $1-P\left(r_{1} \mid s_{1}\right)$. However, this last condition is violated by much of the data on signal detection because these data show that, when the signal $\left(s_{1}\right)$ is very frequent, both the hit and false-alarm rates are close to 1 . For this reason, this modification cannot account for the data of Dusoir and of Creelman and Donaldson.

\section{Model III}

The last modification we will consider is a pure error-correcting model in which the probability of a criterion shift after an error is an increasing function of (a) the difference between the sensory information, $X$, and the criterion, c, on that trial, and (b) the magnitude of the (symmetric) payoffs. It has already been shown (Thomas, 1973, Section 4) that, when certain conditions are put on the distribution of $X$, if and only if $\pi \geqslant 1 / 2$,

$$
\frac{\mathrm{P}\left(\mathrm{r}_{1} \mid \mathrm{s}_{2}\right)}{1-\mathrm{P}\left(\mathrm{r}_{1} \mid \mathrm{s}_{1}\right)} \leqslant \frac{\pi}{1-\pi},
$$

implying that $\mathrm{P}\left(\mathrm{r}_{1}\right) \leqslant \pi$. Further, the effect of increasing payoff magnitude is to move $P\left(r_{1}\right)$ closer to $\pi$. This modification also allows us to discuss the effect of varying signal strength, and we now do so.

Let the probability density function of the sensory information $X$ be $f(x)$ when $s_{2}$ is presented and $f(x-\mu)$, $\mu \geqslant 0$, when $s_{1}$ is presented. Let $R(x)=\int_{x}^{\infty} f(y) d y$, and the criterion shift probabilities be $p_{12}=p(X-c)$ and $p_{21}=p(c-X)$, where $p(0)=0$ and $p^{\prime}(x) \geqslant 0$. We assume that $f(x)=f(-x)$ and that $-\log f(x)$ is convex (these conditions are satisfied by the normal and logistic distributions). It has been shown previously that, neglecting terms $o\left(\Delta^{1 / 2}\right)$, the stationary mean criterion value $\mathrm{c}$ satisfies

$$
\begin{aligned}
g(c, \mu) \equiv & (1-\pi) \int_{0}^{\infty} \mathrm{p}^{\prime}(\mathrm{x}) \mathrm{R}(\mathrm{x}+\mathrm{c}) \mathrm{dx} \\
& -\pi \int_{0}^{\infty} \mathrm{p}^{\prime}(\mathrm{x}) \mathrm{R}(\mathrm{x}+\mu-\mathrm{c}) \mathrm{dx}=0
\end{aligned}
$$

From Equation 4, we get

$$
\begin{aligned}
\frac{d c}{d \mu} & =\frac{-\partial g / \partial \mu}{\partial g / \partial c} \\
& =\frac{\pi \int_{0}^{\infty} p^{\prime}(x) f(x+\mu-c) d x}{\pi \int_{0}^{\infty} p^{\prime}(x) f(x+\mu-c) d x+(1-\pi) \int_{0}^{\infty} p^{\prime}(x) f(x+c) d x}
\end{aligned}
$$

Now it can be shown that $c \leqslant \mu / 2$ if and only if $\pi \geqslant 1 / 2$, and that the conditions on $f(x)$ imply that

$$
\frac{d}{d c} \frac{f(x+c)}{f(c)} \leqslant 0 \quad \text { if } x \geqslant 0 .
$$

Therefore, if $c \leqslant \mu / 2, \mu-c \geqslant \varepsilon$ and

$$
\frac{\mathrm{f}(\mathrm{x}+\mu-\mathrm{c})}{\mathrm{f}(\mu-\mathrm{c})} \leqslant \frac{\mathrm{f}(\mathrm{x}+\mathrm{c})}{\mathrm{f}(\mathrm{c})}
$$

implying, since $p^{\prime}(x) \geqslant 0$, that

$$
\frac{\int_{0}^{\infty} p^{\prime}(x) f(x+\mu-c)}{\int_{0}^{\infty} p^{\prime}(x) f(x+c)} \leqslant \frac{f(\mu-c)}{f(c)}
$$


Therefore, if $\pi>1 / 2$, using the symmetry of $f(x)$,

$$
\frac{\mathrm{dc}}{\mathrm{d} \mu} \leqslant \frac{\pi \mathrm{f}(\mathrm{c}-\mu)}{\pi \mathrm{f}(\mathrm{c}-\mu)+(1-\pi) \mathrm{f}(\mathrm{c})}
$$

The frequency of $r_{1}$ responses is given by

$$
\begin{gathered}
\mathrm{P}\left(\mathrm{r}_{1}\right)=\pi \mathrm{R}(\mathrm{c}-\mu)+(1-\pi) \mathrm{R}(\mathrm{c}) . \\
\frac{\mathrm{dP}\left(\mathrm{r}_{1}\right)}{\mathrm{d} \mu}=\frac{\partial \mathrm{P}\left(\mathrm{r}_{1}\right)}{\partial \mu}+\frac{\partial \mathrm{P}\left(\mathrm{r}_{1}\right)}{\partial \mathrm{c}} \frac{\mathrm{dc}}{\mathrm{d} \mu} \\
=\pi \mathrm{f}(\mathrm{c}-\mu)-[\pi \mathrm{f}(\mathrm{c}-\mu)+(1-\pi) \mathrm{f}(\mathrm{c})] \frac{\mathrm{dc}}{\mathrm{d} \mu} \\
\geqslant 0, \text { if } \pi \geqslant 1 / 2 \text { (on using Equation 5). }
\end{gathered}
$$

In other words, for this generalization of the error-correcting model, $P\left(r_{1}\right)$ is less than $\pi$ if and only if $\pi \geqslant 1 / 2$, and, as signal strength increases, $P\left(r_{1}\right)$ approaches $\pi$.

\section{EXPERIMENTS WITHOUT FEEDBACK}

In the pure error-correcting model, the criterion $\mathrm{c}$ decreases only if $X$ on that trial is less than $c$ (and $s_{1}$ is the stimulus). Therefore, if $c$ changes, it does so in the direction of $X$. This assumption about criterion adjustment is common to all models that assume the subject computes a standard as a weighted average of the most recent sensory information. We shall retain this assumption in our consideration of two models that are applicable to experiments without feedback. We also assume that the density function $\mathrm{f}(\mathrm{x})$ is symmetric about 0.

\section{Model IV}

In the first of these models, we assume that the shift, $\Delta c$, in criterion is given by

$$
\begin{aligned}
\Delta c & =\Delta \text { with probability } p_{1} \text { if } X \geqslant c \\
& =-\Delta \text { with probability } p_{2} \text { if } X<c,
\end{aligned}
$$

where $\Delta>0$. Proceeding as in the proof of Theorem 2.1 (Thomas, 1973), it can be shown that, neglecting terms $o\left(\Delta^{1 / 2}\right)$, the stationary mean criterion value $c$ satisfies

$$
\begin{aligned}
0= & \pi\left[p_{1} R(c-\mu)-p_{2} F(c-\mu)\right] \\
& +(1-\pi)\left[p_{1} R(c)-p_{2} F(c)\right],
\end{aligned}
$$

that is, putting $\alpha=\mathrm{p}_{1} / \mathrm{p}_{2}$,

$$
\frac{\mathrm{F}(\mathrm{c})-\alpha \mathrm{R}(\mathrm{c})}{\alpha \mathrm{R}(\mathrm{c}-\mu)-\mathrm{F}(\mathrm{c}-\mu)}=\frac{\pi}{1-\pi} \text {. }
$$

Probability matching requires that $c$ also satisfy

$$
\frac{\mathrm{R}(\mathrm{c})}{\mathrm{F}(\mathrm{c}-\mu)}=\frac{\pi}{1-\pi} \text {. }
$$

It is easily seen that Equations 7 and 8 are simultaneously satisfied if

$$
\alpha=\frac{\mathrm{F}(\mathrm{c}-\mu)}{\mathrm{R}(\mathrm{c})}=\frac{1-\pi}{\pi} .
$$

In other words, the model in Equation 6 generates probability matching if the conditioning probabilities, $\mathrm{p}_{\mathrm{i}}$, satisfy $\left(\mathrm{p}_{1} / \mathrm{p}_{2}\right)=(1-\pi) / \pi$.

In order to account for deviations from matching, one has to suppose that $\alpha \neq(1-\pi) / \pi$, in general. For example, suppose that $\alpha=1$ for all values of $\pi$, and that $f(x)=f(-x)$. Then the left-hand sides of Equations 7 and 8 are increasing and decreasing functions, respectively, of $c$, and equal 1 when $c=\mu / 2$. We now have that $\pi \geqslant 1 / 2$ implies that $\pi /(1-\pi) \geqslant 1$, which, from Equation 7 with $\alpha=1$, implies that $c \geqslant \mu / 2$, which implies that $\mathrm{R}(\mathrm{c}) / \mathrm{F}(\mathrm{c}-\mu) \leqslant 1 \leqslant \pi /(1-\pi)$. This last inequality (cf. Equation 1) implies that $P\left(r_{1}\right) \leqslant \pi$, which is indicated in Dusoir's (1974) data.

So far, this model has remained open with respect to the determinants of $\alpha$. We have already shown that, if $\alpha$ depends on the experimenter-determined $\pi$ in the manner of Equation 9, probability matching results. Whether or not $\alpha$ depends on $\pi$, we need to specify how it might depend on payoff magnitude, and how $\mathrm{P}\left(\mathrm{r}_{1}\right)$ might vary with $\alpha$. With regard to the latter, on differentiating Equation 7 with $\pi$ fixed, we see that $\mathrm{dc} / \mathrm{d} \alpha>0$, which implies that $\mathrm{P}\left(\mathrm{r}_{1}\right)$ is inversely related to $\alpha$. With regard to the former, it seems reasonable to suppose that a decrease in $\alpha$ corresponds to an increase in the subject's bias towards the $r_{1}$ response, partly because a decrease in $p_{1} / p_{2}=\alpha$ seems, in the small, to correspond to an increase in the bias towards reducing the criterion, and partly because, in the large, the stationary value $c$ does decrease as $\alpha$ decreases.

A simple method of defining "bias towards $\mathrm{r}_{\mathbf{1}}$ " as a function of symmetric payoffs and of $\pi$ is to say that bias is directly related to the difference in expected payoff between responding $r_{1}$ and responding $r_{2}$ without paying attention to the sensory information. That is, if $A$ is the gain $(G)$ for a correct decision and -B is the gain for an incorrect decision, and if the subject were to respond without regard to $X$, then bias is directly related to

$$
\begin{aligned}
\mathrm{E}\left(\mathrm{G} \mid \mathrm{r}_{1}\right)- & \mathrm{E}\left(\mathrm{G} \mid \mathrm{r}_{2}\right) \\
& =[\pi \mathrm{A}-(1-\pi) \mathrm{B}]-[(1-\pi) \mathrm{A}-\pi \mathrm{B}] \\
& =(2 \pi-1)(\mathrm{A}+\mathrm{B}) .
\end{aligned}
$$


With this definition, "bias towards $r_{1}$ " exists even if the payoffs are symmetric, provided $\pi \neq 1 / 2$. For fixed $\pi>1 / 2$, as $(A+B)$ increases, bias towards $r_{1}$ increases, implying that $\alpha$ decreases, which implies that $P\left(r_{1}\right)$ increases.

Dusoir (Note 1) ran two groups of subjects, both having $\pi=.8$, but $(\mathrm{A}+\mathrm{B})$ was 1 for one group and was 10 for the other. When $A+B$ was $1, P\left(r_{1}\right)=.755$ and when $A+B$ was $10, P\left(r_{1}\right)=.771$. These results are consistent with the relation between $\alpha$ and payoffs assumed above.

Another issue which we should address is the possible dependence of $\mathrm{P}\left(\mathrm{r}_{1}\right)$ on $\mu$. We can proceed as in the derivation of Equation 5 to see that, if $c$ is given by Equation 7,

$$
\frac{\mathrm{dc}}{\mathrm{d} \mu}=\frac{\pi \mathrm{f}(\mathrm{c}-\mu)}{\pi \mathrm{f}(\mathrm{c}-\mu)+(1-\pi) \mathrm{f}(\mathrm{c})},
$$

which implies that $\mathrm{P}\left(\mathrm{r}_{1}\right)$ is independent of $\mu$. Below, we will consider some of Dusoir's (1974) data that are relevant to this prediction.

\section{Model V}

The second model we shall consider in this section is similar to that used in deriving Equation 4. Here we assume that the criterion shift $\Delta \mathrm{c}$ is given by

$$
\Delta \mathrm{c}=\delta(\mathrm{X}-\mathrm{c})
$$

where $\delta(x)$ is a bounded, increasing function of $\mathrm{x}$, and $\delta(\mathrm{x})=-\delta(-\mathrm{x})$. The stationary value $\mathrm{c}$ satisfies

$$
\begin{aligned}
0 & =E(\Delta c) \\
& =\pi \int_{-\infty}^{\infty} f(x-\mu) \delta(x-c) d x+(1-\pi) \int_{-\infty}^{\infty} f(x) \delta(x-c) d x .
\end{aligned}
$$

On integrating by parts and using the assumed properties of $\delta(\mathrm{x})$, we get

$$
\frac{\int_{0}^{\infty} \delta^{\prime}(x)[F(c+x)-R(c-x)] d x}{\int_{0}^{\infty} \delta^{\prime}(x)[R(c-\mu-x)-F(c-\mu+x)] d x}=\frac{\pi}{1-\pi}
$$

Equation 7 with $\alpha=1$ is a special case of Equation 11. It is not difficult to show that the left-hand side of Equation 11 is an increasing function of $c$ and equals 1 when $c=\mu / 2$. Hence, as argued in the case of Model IV, $\pi \geqslant 1 / 2$ if and only if $P\left(r_{1}\right) \leqslant \pi$.

To see the dependence of $P\left(r_{1}\right)$ on $\mu$, we could try to establish the Inequality 5 for this model. However, it can be shown that Inequality 5 is not necessarily satisfied, and the sign of $\mathrm{dP}\left(\mathrm{r}_{1}\right) / \mathrm{d} \mu$ is indeterminate unless extra assumptions are introduced. Because of the similarity between this model and Model III, a reasonable conjecture is that, for the ranges of $c$ and $\mu$ found in most studies, $\mathrm{P}\left(\mathrm{r}_{1}\right)$ increases with $\mu$ if $\pi \geqslant 1 / 2$.

Dusoir's (1974) data allow us to look at the dependence of $\mathrm{P}\left(\mathrm{r}_{1}\right)$ on $\mu$ if it is assumed that $\mathrm{d}^{\prime}$ is a constant multiple of $\mu$. In his Experiment II, 24 subjects were run in a low $(\mathrm{L})$ and a high $(\mathrm{H})$ discriminability condition and $\pi=.8$ in both conditions. Of the 24 subjects, 15 had a $\mathbf{P}\left(\mathrm{r}_{1}\right)$ of .8 or less in both conditions, as required by Model IV with $\alpha=1$. Of these 15 subjects, 9 show an increase in $\mathrm{P}\left(\mathrm{r}_{1}\right)$ as discriminability is increased (see Dusoir, 1974, Table 2).

Another way of examining the dependence of $P\left(r_{1}\right)$ on $\mu$ is to correlate $\mathrm{P}\left(\mathrm{r}_{1}\right)$ and $\mathrm{d}^{\prime}$ across subjects. If subjects differ only with respect to $\mu$, then this correlation should be positive if $\mathrm{P}\left(\mathrm{r}_{1}\right)$ increases as $\mu$ increases. Using the data of those subjects for which $\mathrm{d}^{\prime}$ is defined and positive (Dusoir, 1974, Table 2), the correlation between $\mathrm{P}\left(\mathrm{r}_{1}\right)$ and $\mathrm{d}^{\prime}$ is .38 for condition $\mathrm{L}$ and .55 for condition $H$. In his Experiment $I$, this correlation, computed from the data in Table 1 , is only .13. Thus the data from Experiment II offer some support for the conjecture that, if Model $\mathrm{V}$ is valid, then $\mathrm{P}\left(\mathrm{r}_{1}\right)$ is an increasing function of $\mu .^{1}$

\section{SUMMARY}

In some detection and recognition studies, response frequencies are not as extreme (that is, distant from $1 / 2$ ) as the corresponding stimulus frequencies. A distinction can be made between those studies in which trial-by-trial feedback is given to the subject, and those studies without feedback. When feedback is given, assumptions can be made about the effects of feedback on criterion adjustment, for example, the assumptions made in Models I to III. It has been shown that certain observed deviations from probability matching can be accounted for by at least two versions of an error-correcting model, but not by a model that permits criterion adjustment after correct responses. One version of the error-correcting model allows us to specify the dependence of response frequency on payoff magnitude and on signal strength.

When feedback is not given, criterion adjustment (if there is any) can be based only on the responses made and on the sensory information $X$ obtained by the subject. In Models IV and $V$, it was assumed that adjustment depends on the deviation between the sensory information and the criterion on the current trial. In Model IV, criterion setting is affected by stimulus probability $\pi$ and payoffs by means of the parameter $\alpha$, which equals the ratio of the upward and downward step sizes. For this model, probability matching requires that $\alpha=(1-\pi) / \pi$; when $\alpha=1$, response frequencies are less extreme than corresponding stimulus frequencies; and it was suggested that when $\pi>1 / 2, \alpha$ might be inversely related to the sum of the 
pay off magnitudes A and B. Also, response frequency is independent of signal strength. In Model V, the criterion shifts towards $X$ by an amount directly related to the distance of $\mathrm{X}$ from the criterion. This model accounts for some of the observed deviations from matching, and it was conjectured that the frequency of reporting the more frequent stimulus $\left[P\left(r_{1}\right)\right.$, if $\left.\pi>1 / 2\right]$ increases as signal strength increases. Existing data provide some support for this conjecture.

\section{REFERENCE NOTE}

1. Dusoir, A. E. Thomas and Legge's matching hypothesis applied to a detection task without feedback. Manuscript submitted for publication. 1975.

\section{REFERENCES}

Creelman, C. F., \& Donaldson, W. ROC curves for discrimination of linear extent. Joumal of Experimental Psychology, 1968. 77. 514-516.

Dorfman, D. C.. \& Biderman, M. A learning model for a continuum of sensory states. Journal of Mathematical Psychology, 1971, 8, 264-284.

Dorfman, D. D., Saslow, C. F., \& Simpson, J. C. Learning models for a continuum of sensory states reexamined. Journal of Mathematical Psychology, 1975, in press.
Dusoir, A. E. Thomas and Legge's matching hypothesis for detection and recognition tasks: Two tests. Perception \& Psychophysics, 1974, 16. 466-470.

KAC, M. A note on learning signal detection. IRE Transactions on Information Theory, 1962, 8, 126-128.

Norman, M. F. Markov processes and learning models. New York: Academic Press, 1972.

PARKs, T. E. Signal-detectability theory of recognition memory performance. Psychological Review, 1966, 73, 44-58.

Thomas, E. A. C. On a class of additive learning models: Error-correcting and probability matching. Journal of Mathematical Psychology, 1973, 10, 241-264.

Thomas, E. A. C., \& Legge. D. Probability matching as a basis for detection and recognition decisions. Psychological Review, 1970, 77, 65-72.

\section{NOTE}

1. It may be that the correlation between $P\left(r_{1}\right)$ and $d^{\prime}$ is due partly to the dependence of each on $P\left(r_{1} \mid s_{1}\right)$ and $P\left(r_{1} \mid s_{2}\right)$. A useful "null hypothesis" is that $P\left(r_{1} \mid s_{1}\right)$ and $P\left(r_{1} \mid s_{2}\right)$ are independent random variables. Under this hypothesis, the correlation between $P\left(r_{1}\right)$ and $d^{\prime}$ can be positive or negative depending on the mean and variance of $P\left(r_{1} \mid s_{1}\right)$ and $P\left(r_{1} \mid s_{2}\right)$, so that this null hypothesis alone is not sufficient to account for a positive correlation between $\mathbf{P}\left(r_{1}\right)$ and $d^{\prime}$.

(Received for publication January 27, 1975; revision accepted May 12, 1975.) 